\title{
Effects of the Inhalational Anesthetics Halothane and Sevoflurane on an Experimental Model of Hepatic Injury
}

\author{
Andrea Fogaça Soubhia 1, Susi Lauz 2, Edna Frasson de Souza Montero ${ }^{3}$, Alessandro Menezes 4 , \\ Luciane Bicca Mespaque ${ }^{5}$, Emilio Facin ${ }^{5}$
}

Summary: Soubhia AF, Lauz S, Montero EFS, Menezes A, Mespaque LB, Facin E - Effects of the Inhalational Anesthetics Halothane and Sevoflurane on an Experimental Model of Hepatic Injury.

Background and objectives: Hepatic injury after inhalational anesthesia is controversial. Studies have suggested that inhalational agents generate an immune response that can provoke hepatic injury. The objective of this study was to analyze the effects of the inhalational agents halothane and sevoflurane on the liver of rats submitted to hypoxia and reperfusion.

\begin{abstract}
Methods: Thirty Wistar rats, pretreated with $0.1 \%$ phenobarbital for 5 days, with discontinuation of the drug 24 hours before the experiment to cause hepatic injury, were used. Animals were distributed in five groups of six rats each. The control group (C) did not receive any treatment; in the $\mathrm{F}$ group, phenobarbital was used to induce hepatic injury; the Hypoxia group was submitted to $14 \%$ oxygen $\left(\mathrm{O}_{2}\right)$; the $\mathrm{H}$ group received $1 \%$ halothane and $14 \% \mathrm{O}_{2}$; and the $\mathrm{S}$ group received $2 \%$ sevoflurane and $14 \% \mathrm{O}_{2}$. Twenty-four hours after exposure to the gases, blood samples were collected to evaluate transaminases (AST and ALT), and liver samples were collected for histological evaluation. Kruskal-Wallis Analysis of Variance and the Newman-Keuls test were used.
\end{abstract}

Results: Enzymatic activity mean values of AST (280.33, for halothane, 181, for sevoflurane) and ALT (235 for halothane, and 48.33 , for sevoflurane) did not show significant differences, and all groups showed elevated values. Compared to halothane on optical microscopy, sevoflurane had lower indices of morphologic changes with $p=0.045$, for steatosis, $p=0.0075$, for inflammatory infiltrate, and $p=0.0074$, for necrosis.

Conclusions: Compared to the halothane group, sevoflurane did not show injuries of the liver parenchyma on optical microscopy.

Keywords: Halothane; General Anesthesia; Inhalational Anesthetics; Hepatopathies.

\section{INTRODUCTION}

Complex liver surgical procedures, like extensive resections, transplants, and trauma, include most of the time a temporary occlusion of the hepatic pedicle. Patients with advanced liver disease (borderline liver function) who undergo large size surgeries have an extremely high postoperative morbidity and mortality ${ }^{1,2}$. Hypoxia caused by occlusion of the pedicle or by a significant reduction in hepatic blood flow triggers a process of ischemic injury that intensifies with reperfusion of the liver, compromising not only this organ, but also others related.

Received from Universidade Federal do Rio Grande (FURG), Brazil.

1. Health Sciences Professor at FURG; Assisting Professor of Faculdade de Medicina da FURG

2. Professor, Physician; Associated Professor of Faculdade de Medicina da FURG

3. Professor, Physician; Professor of Surgery Department of the Universidade Federal de São Paulo (UNIFESP)

4. Professor of Ciências da Saúde; Professor of Faculdade da Medicina da FURG

5. Medical Student; Medical Student of Faculdade de Medicina da FURG

Submitted on November 27, 2010.

Approved on July 25, 2011.

Correspondence to:

Dra. Andrea Fogaça Soubhia

Rua General Portinho 35/ apto 803

96200210 - Rio Grande, RS, Brazil

E-mail: andsoubhia@yahoo.com.br
The role of inhalational anesthetics in the genesis of the ischemic process during surgery has been investigated to determine the degree of their involvement on the pathophysiology of the ischemic injury. Those investigations are aimed at the development of anesthetic alternatives to minimize the local and systemic repercussions of the ischemic injury.

Inhalational anesthetics are the most commonly used agents for maintenance of general anesthesia. The popularity of those drugs to establish anesthesia is based on a range of attractive, such as ease of administration, previsibility of their effects, low cost, and extensive training of anesthesiologists. However, every drug has side effects and among them hepatic injury and the associated high mortality. Studies have been conducted to establish the precise pathophysiology of this injury and factors and agents involved in its genesis ${ }^{3-9}$.

Most hypotheses on the mechanisms of action of inhalational anesthetics are based on their physical-chemical characteristics and biochemical and neurophysiological effects, besides proposing the cellular membrane, both in its lipidic and protein portions, as sites of action ${ }^{5}$.

Halothane seems to be the agent associated with hepatic cell injury due to the binding of its oxidative metabolites to hepatic cytochromes - they behave as haptens, inducing hypersensitivity responses. The oxidative metabolic pathway involving cytochrome P450 during exposure to halothane is identical to the metabolic pathway observed with enflurane, 
isoflurane, and desflurane. However, expression of neoantigens should be related to the amount of metabolism of each agent. This suggests that, regarding the antigenic load, halothane $>$ enflurano $>$ isoflurane $>$ desflurane, in a proportion related to halothane as of 10,100 , and 1.000 times less, respectively. Sevoflurane is not metabolized to trifluoroacetyl halogenate but to hexafluoroisopropanol that does not behave like a neoantigen. But cases of hepatitis after exposure to sevoflurane have been reported, which might indicate more than one mechanism involved in hepatic injury or the presence or cross reaction, since, in the cases reported, patients had previous contact with other inhalational anesthetics. The objective of this study was to investigate the effects of the inhalational anesthetics halothane and sevoflurane in an experimental model of hepatic injury ${ }^{10-12}$.

\section{METHODOLOGY}

\section{Population}

Thirty male Wistar rats (Rattus norvegicus albinus) with mean weight of $350 \mathrm{~g}$ and 3 months of age were used. Animals, from the controlled conventional laboratory of the Universidade Federal do Rio Grande (FURG), were operated in the Laboratório de Morfologia Experimental of the department of Cirurgia Geral of FURG. The investigation was evaluated and approved by the Ethics on Research Committee of FURG, \# 21/2007. The number of animals was defined according to the norms of the Colégio Brasileiro de Experimentação Animal (COBEA) that determines 6 animals per group as the minimum significant number.

The sample was randomly distributed in five groups of 6 rats each, as described: Control Group $(n=6)$; Phenobarbital Group $(n=6)$; Hypoxia Group $(n=6)$; Halothane Group $(n=6)$; and Sevoflurane Group $(n=6)$.

\section{Experimental Procedure}

All animals, except for the Control Group, were pretreated with $0.1 \%$ phenobarbital $\left(1 \mathrm{mg} \cdot \mathrm{mL}^{-1}\right)$, added to their water during 5 days, to induce the $\mathrm{P}-450$ complex, with a minimal dose of $15 \mathrm{mg}^{\text {day }}{ }^{-1}$ of phenobarbital per rat, which was discontinued 24 hours before the experiment. Animals that did not achieve this index were discarded (control performed by the minimum ingestion of $15 \mathrm{~mL}$.day ${ }^{-1}$ of water).

Animals in the Hypoxia, Halothane, and Sevoflurane Groups were individually placed in glass cages connected to an anesthesia device with calibrated vaporizers. Rats received a mixture of $14 \%$ oxygen and $86 \%$ nitrogen through the anesthesia device via a flow meter. The mixture caused hypoxia at which point the anesthetics were administered:

Hypoxia Group: animals received only the mixture of $14 \%$ oxygen and $86 \%$ nitrogen during 2 hours.
Halothane Group: Animals received a mixture of $14 \%$ oxygen and $86 \%$ nitrogen plus $1 \%$ halothane (via a vaporizer calibrated for this concentration) during a 2-hour period.

Sevoflurane Group: Animals received a mixture of $14 \%$ oxygen and $86 \%$ nitrogen plus $2 \%$ sevoflurane (via a vaporizer calibrated for this concentration) during a 2-hour period.

At the end of the anesthetic procedure, rats were returned to their individual cages and received food and water $a d$ libitum.

\section{Material collection and euthanasia}

The Control and Phenobarbital groups were not exposed to previous inhalational anesthesia, but they underwent collection of material and death along with the other groups, i.e., 24 hours after the procedure in the Hypoxia, Halothane, and Sevoflurane groups.

The blood collected was sent immediately to the Laboratório Central of Hospital Universitário of FURG. Enzymatic activities of aspartate aminotransferase (AST) and alanine aminotransferase (ALT) were measured by a kinetic method in an autoanalyzer device (Selectra).

Five samples were obtained from each liver for histological exam by optical microscopy. Samples were fixed in $10 \%$ tamponated formaldehyde and were stained with hematoxylineosin (HE) before histological evaluation. In the liver, the occurrence of the following was analyzed:

1 - Microvesicular steatosis: Defined as the presence of lipid accumulation of microvesicles in the cytoplasm whose volume is lower than the nucleus;

2 - Leukocyte infiltrate: Presence of leukocytes (especially neutrophils) in different areas of the hepatic lobule;

3 -Necrosis: Characterized by condensation or obliteration of the nucleus, intense cytoplasmic eosinophilia, and destruction and architectural loss of hepatocyte cords (hepatocellular detrabeculation);

4 -Apoptosis: Characterized by the aspect of the nucleus, i.e., the pattern of chromatin in the nucleus and aspect of the nuclear cytoplasm.

Results regarding AST and ALT were expressed as means and standard deviation, while the results of histological analysis as medians and quartiles. Kruskal-Wallis Analysis of Variance was used to analyze the enzymatic activity and histological changes, and the Newman-Keuls test was used to compare means. It was considered a level of significance of $5 \%$. The analytical study was performed by the Statistics Department of FURG using the Bioestat 4.0 software.

\section{RESULTS}

Regarding the values of aspartate aminotransferase (AST) in rats in the Control, Phenobarbital, Hypoxia, Halothane, and Sevoflurane Groups, the following results were observed (Table I). 
Table I - Comparative Values of AST in the Study Groups

\begin{tabular}{|c|c|c|c|c|c|}
\hline \multicolumn{6}{|l|}{ AST } \\
\hline & Control & Phenobarbital & Hypoxia & Halothane & Sevoflurane \\
\hline & 100 & 110 & 170 & 358 & 196 \\
\hline & 119 & 123 & 133 & 214 & 196 \\
\hline & 120 & 121 & 155 & 219 & 185 \\
\hline & 130 & 109 & 154 & 284 & 188 \\
\hline Mean Value & 117.50 & 117.50 & 148.50 & 280.33 & 181.00 \\
\hline Standard-deviation & 10.67 & 6.28 & 13.98 & 60.67 & 20.57 \\
\hline Variation Coefficient - VC (\%) & $9.08 \%$ & $5.34 \%$ & $9.41 \%$ & $21.64 \%$ & $11.36 \%$ \\
\hline
\end{tabular}

Kruskal-Wallis Analysis of Variance test. $\mathrm{H}_{\text {CRITICAL }}=9.49 ; \mathrm{H}_{\text {CALCULATED }}=25.94 ; \mathrm{p}=0.0000$; comparison of means by the Newman-Keuls test $\mathrm{p}<0.05$;

Control $=$ Phenobarbital $=$ Hypoxia.

According to the statistical analysis, the levels of aspartate aminotransferase (AST) in the Control Group were similar to those in the Phenobarbital and Hypoxia Groups; however, higher levels were observed in the Halothane and Sevoflurane Groups without significant differences between both groups.

As for the levels of alanine aminotransferase (ALT) in rats in the Control, Phenobarbital, Hypoxia, Halothane, and Sevoflurane Groups the results are shown in Table II.

On statistical analysis, the levels of alanine aminotransferase (ALT) in the Control Group were similar to those in the Phenobarbital and Hypoxia Groups; however, higher levels were observed in the Halothanes and Sevoflurane Groups, without significant differences between them.

Table III shows the results for hepatic microvesicular steatosis observed on optical microscopy stained by $\mathrm{HE}$ in rats in the Control, Phenobarbital, Hypoxia, Halothane, and Sevoflurane Groups.

On statistical analysis, the levels of hepatic microvesicular steatosis in the Control Group were similar to the Phenobarbital, Hypoxia, and Sevoflurane Groups; higher statistically significant levels were observed in the Halothane Group.
Regarding the hepatic inflammatory infiltrate observed in optical microscopy stained by HE, the results are presented in Table IV.

On statistical analysis, the hepatic inflammatory infiltrate in the Control Group was similar to that observed in the Phenobarbital, Hypoxia, and Sevoflurane Groups; however, higher statistically significant levels were observed in the Halothane Group.

As for liver necrosis observed on optical microscopy stained by HE in rats in the Control, Phenobarbital, Hypoxia, Halothane, and Sevoflurane Groups, the results are presented in Table $\mathrm{V}$.

On statistical analysis, the levels of hepatic in the Control Group were similar in the Phenobarbital, Hypoxia, and Sevoflurane Groups; however, higher statistically significant levels were observed in the Halothane Group.

Table VI show liver apoptosis observed on optical microscopy stained by HE in the Control, Phenobarbital, Hypoxia, Halothane, and Sevoflurane Groups.

On statistical analysis, the levels of liver apoptosis in the Control Group were similar to those observed in the Pheno-

Table II - Comparative Values of ALT in the Study Groups

\begin{tabular}{|c|c|c|c|c|c|}
\hline \multicolumn{6}{|l|}{ ALT } \\
\hline & Control & Phenobarbital & Hypoxia & Halothane & Sevoflurane \\
\hline & 20 & 20 & 44 & 395 & 50 \\
\hline & 21 & 29 & 41 & 176 & 53 \\
\hline & 24 & 28 & 47 & 174 & 50 \\
\hline & 32 & 42 & 50 & 135 & 39 \\
\hline Mean & 25.00 & 29.67 & 44.33 & 235.83 & 48.33 \\
\hline Standard Deviation & 4.90 & 9.69 & 5.65 & 129.05 & 4.97 \\
\hline Variation Coefficient - VC (\%) & $19.60 \%$ & $32.66 \%$ & $12.75 \%$ & $54.72 \%$ & $10.21 \%$ \\
\hline
\end{tabular}

Kruskal-Wallis Analysis of Variance test. $\mathrm{H}_{\text {CRITICAL }}=9.49 ; \mathrm{H}_{\text {CALCULATED }}=24.16 ; p=0.0001^{*} ;$ comparison of means by the Newman-Keuls test $p<0.05$;

Control $=$ Phenobarbital $=$ Hypoxia . 
Table III - Analysis of Microvesicular Steatosis in the Study Groups

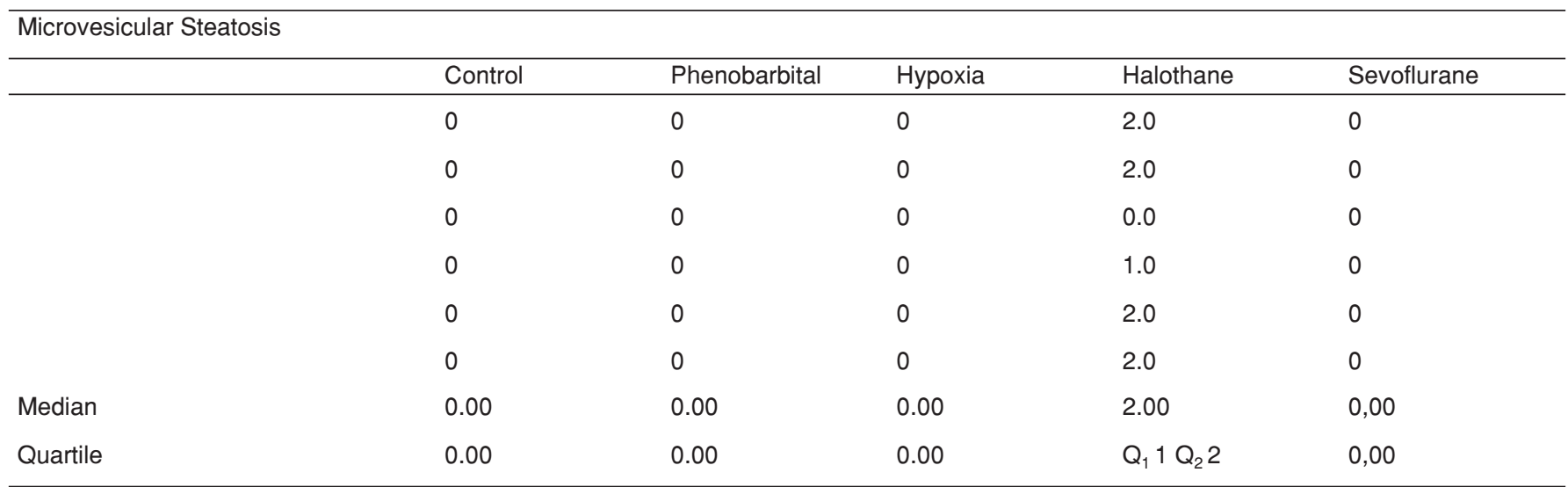

Kruskal-Wallis Analysis of Variance test. $\mathrm{H}_{\text {CRITICAL }}=9.49 ; \mathrm{H}_{\text {CALCULADO }}=9.6990 ; p=0.0458^{*} ;$ comparison of means by the Newman-Keuls test $p<0.0139^{*}$; Control $=$ Phenobarbital $=$ Hypoxia $=$ Sevoflurane Halothane .

Table IV - Analysis of the Inflammatory Infiltrate in the Study Groups

\begin{tabular}{|c|c|c|c|c|c|}
\hline \multicolumn{6}{|c|}{ Inflammatory Infiltrate } \\
\hline & Control & Phenobarbital & Hypoxia & Halothane & Sevoflurane \\
\hline & 0 & 0 & 0 & 1.0 & 0 \\
\hline & 0 & 0 & 0 & 3.0 & 0 \\
\hline & 0 & 0 & 0 & 2.0 & 0 \\
\hline & 0 & 0 & 0 & 1.0 & 0 \\
\hline Median & 0.00 & 0.00 & 0.00 & 2.00 & 0.00 \\
\hline Quartile & 0.00 & 0.00 & 0.00 & $Q_{1} 1 Q_{2} 2$ & 0.00 \\
\hline
\end{tabular}

Kruskal-Wallis Analysis of Variance test. $\mathrm{H}_{\text {CRITICAL }}=9.49 ; \mathrm{H}_{\text {CALCULATED }}=13.9510 ; p=0.0075^{*}$; comparison of means by the Newman-Keuls test $p<0.0032^{*}$; Control $=$ Phenobarbital $=$ Hypoxia $=$ Sevoflurane Halothane

Table V - Analysis of Necrosis in the Study Groups

\begin{tabular}{|c|c|c|c|c|c|}
\hline \multicolumn{6}{|l|}{ Necrosis } \\
\hline & Control & Phenobarbital & Hypoxia & Halothane & Sevoflurane \\
\hline & 0 & 0 & 0 & 1,0 & 0 \\
\hline & 0 & 0 & 0 & 3,0 & 0 \\
\hline & 0 & 0 & 0 & 3,0 & 0 \\
\hline & 0 & 0 & 0 & 3,0 & 0 \\
\hline Median & 0.00 & 0.00 & 0.00 & 3.00 & 0.00 \\
\hline Quartile & 0.00 & 0.00 & 0.00 & $Q_{1} 1 Q_{2} 3$ & 0.00 \\
\hline
\end{tabular}

Kruskal-Wallis Analysis of Variance test. $\mathrm{H}_{\text {CRITICAL }}=9.49 ; \mathrm{H}_{\text {CALCULATED }}=13.9697 ; p=0.0074^{*} ;$ comparison of means by the Newman-Keuls test $p<0.0032^{*}$; Control $=$ Phenobarbital $=$ Hypoxia $=$ Sevoflurane Halothane . 
Table VI - Analysis of Apoptosis in the Study Groups

\begin{tabular}{llllll}
\hline APOPTOSIS & & & & \\
\hline & Control & Phenobarbital & Hypoxia & Halothane & Sevoflurane \\
\hline & 0 & 0 & 0 & 0 & 0 \\
& 0 & 0 & 0 & 0 & 0 \\
& 0 & 0 & 0 & 0 & 0 \\
& 0 & 0 & 0 & 0 & 0 \\
Median & 0 & 0 & 0 & 0.00 & 0.00 \\
Quartile & 0 & 0 & 0.00 & 0.00 & 0.00 \\
\hline
\end{tabular}

Kruskal-Wallis Analysis of Variance test. $\mathrm{H}_{\text {CRITICAL }}=9.49 ; \mathrm{H}_{\text {CALCULATED }}=0.0000 ; p=1.0000$; Control $=$ Phenobarbital $=$ Hypoxia $=$ Sevoflurane $=$ Halothane.

barbital, Hypoxia, Sevoflurane, and Halothane Groups without significant difference among them.

\section{DISCUSSION}

In the present study, the experimental model of hepatotoxicity with halogenated anesthetics was used after pretreatment with the enzymatic inductor phenobarbital in the presence of hypoxia. Prior studies ${ }^{3,13}$ made it clear the need of the three factors mentioned to generate hepatic injury. Studies suggest that the liver that has not been pre-induced cannot metabolize halothane sufficiently to trigger the defense mechanisms that cause hepatic tissue injury, even in hypoxic conditions; the lower the oxygen concentration, more the hepatic damage generated by halothane increases ${ }^{3}$. Besides sensitizing the liver to halothane, hypoxia also increases the production of toxic intermediate metabolites of this drug.

When the enzymatic activity - a way of assessing liver function - was submitted to statistical analysis, it did not show a significant difference in the mean values of AST (280.33 for halothane, and 181 for sevoflurane) and ALT (235 for halothane, and 48.33 for sevoflurane). Both groups showed elevated levels, but with higher percentages in the Halothane Group. The findings of enzymatic activity of the present study are in agreement with the data in the literature. Nagata et al. ${ }^{14}$ reported investigations in an experimental model in which sevoflurane produced small transient elevations in the hepatic enzymes, AST and ALT, similar to those resulting from the use of enflurane and halothane. This increase in hepatic enzymes, however, returned to normal levels after 48 hours.

Histological evaluation by optical microscopy allows the differentiation between reversible and irreversible lesions in the hepatic tissue. Liver biopsy has a central role in the evaluation because it represents the most precise manner to determine the nature of the hepatic injury, may it be necrosis, inflammation, steatosis, or fibrosis. In this study, microvesicular steatosis, leukocyte infiltrate, necrosis, and cellular apoptosis were observed in the liver of animals with parameters of parenchymal damage. Microvesicular steatosis is due to the accumulation of fat vacuoles in cells. It is seen as small droplets of fat finely spread in the cytoplasm without dislocating the nucleus, resulting from acute damage promoted by hepatotoxic drugs. Microvesicular steatosis was observed only in the group submitted to treatment with halothane, confirming other studies ${ }^{13}$ in which other isolated treatments with phenobarbital an hypoxia did not generate cellular damage on microscopic analysis. As for sevoflurane steatosis was not observed suggesting that this drug is not toxic for hepatocytes and, therefore, does not generate visible acute damage on optical microscopy.

Regarding the leukocyte infiltrate - especially neutrophils in different areas of the hepatic lobule, characterizing inflammation with oxidative stress and changes in vascular permeability and hepatic edema - the present study showed the same pattern of steatosis, being present only in the Halothane Group.

Cellular necrosis, which manifests by condensation or obliteration of the nucleus by the intense cytoplasmic eosinophilia and destruction and architectural loss of hepatocyte cords (hepatocellular detrabeculation), the mechanism of cellular death in hepatic injury and whether it could evolve in a late phase to apoptosis, is not completely understood. In the data collected for histological analysis by the HE technique, we observed the persistence of the pattern in which necrosis was observed only in the Halothane Group.

In the investigation of apoptosis, which is characterize by the aspect of the nucleus - i.e., by the pattern of chromatin in the nucleus and aspect of the nuclear cytoplasm as a late change of cellular damage evolving to a process of necrosis - this type of cellular destruction was not observed in any groups, since it was an acute experiment.

With similar results to our study, we found in the literature the study of Soma et al. ${ }^{10}$, in which monkeys were anesthetized with sevoflurane during 8 consecutive weeks. At the end of the study no macroscopic, histopathological, or ultrastructural pathologic abnormalities were observed in the liver. 
Although anesthesia-induced hepatitis is not a common occurrence, we should be aware of the association between the disease and the use of halogenated anesthetics. Halothane-induced hepatitis has elevated morbidity and mortality rates and survival of the more affected patients can require a liver transplant. A history of anesthesia-induced hepatitis is a reason to avoid subsequent exposure to halothane or other halogenated anesthetics, since crossed immunity although rare can occur. In summary, avoiding halothane seems to be the most effective isolated way of decreasing the frequency of anesthesia-induced hepatitis. On the other hand, as explained before, sevoflurane is a safe alternative because it is not metabolized as other halogenated anesthetics and it does not form haptens. Current evidence does not indicate the presence of any sevoflurane metabolites that can cause severe hepatic injury ${ }^{11}$, and studies ${ }^{12}$ to correlate its metabolite (compound $A$ ) to hepatotoxicity have been inconclusive.

We conclude that halothane should not be used in adults with prior history of anesthesia-induced hepatic injury and we advise against its use in the pediatric population. Although in children the incidence of hepatitis seems to be very low, one should consider the immunologic memory, which can trigger hepatic injury in adult life after another exposure. Sevoflurane has a low hepatotoxic potential as described in the current medical literature. Such statement corroborates the studies of the present study.

Studies have confirmed that metabolites of sevoflurane are less reactive (and, most likely, cause less damage) than those of halothane, enflurane, isoflurane, and even desflurane ${ }^{12}$. Sevoflurane preserves more efficiently the blood flow and oxygen delivery to the liver than halothane, enflurane, or desflurane; the effects on hepatic perfusion and metabolic function are similar to those of isoflurane ${ }^{15}$. It seems unlikely that sevoflurane can cause clinically important severe postoperative hepatic dysfunction; for this reason, it is the ideal anesthetic in patients with previous hepatic disease. Its use is essential in large size surgeries and liver transplants, intervention in which postoperative live dysfunction could have harmful effects on patients with history of liver damage.

Thus, we conclude that the Sevoflurane Group, compared to the Halothane Group, did not have microscopic damage in the liver parenchyma. 